\title{
Gravierender Mangel an Ärzten im Katastrophengebiet Aceh / Nordsumatra
}

\author{
O. Seeger
}

Am 5. und 7. Januar 2005, 12.45 Uhr, flogen die beiden ersten Delegationen Schweizer Ärzte mit medizinischer Ausrüstung für eine erste Notversorgung von Zürich nach Medan. Sie helfen im Katastrophengebiet Aceh, die dringend nötige medizinische Notversorgung aufzubauen.

Korrespondenz:

Oliver Seeger

PanEco

Chileweg 5

CH-8415 Berg am Irchel

E-Mail: mail@paneco.ch
Dem Aufruf der schweizerischen Umweltstiftung PanEco, welche nach Absprache mit dem Verband Schweizerischer Assistenz- und Oberärzte (Sektion Zürich) in der ganzen Schweiz Ärztinnen und Ärzte und Pflegefachkräfte sucht, sind viele Interessierte gefolgt. Es haben sich bereits nicht nur Schweizer, sondern auch Personen aus Holland, Belgien, Australien, Thailand und Singapur gemeldet, die auch ihre Hilfe anbieten.

Der für die indonesische NR-Umweltorganisation YEL arbeitende Arzt Dr. Sofyan Tan hat berichtet, dass dringend mindestens 500 Ärztinnen und Ärzte und 700 Pflegefachkräfte sowie Medikamente in diesem vom Tsunami am schwersten betroffenen Katastrophengebiet Aceh/Nordsumatra benötigt werden. Dr. Ian Singlet on, der wissenschaftliche Direktor des SCOP, meldete in einem Bericht von grauenhaften Zuständen bezüglich der medizinischen Versorgung der Überlebenden, von Ortschaften, die völlig zerstört und entvölkert sind, und von Gegenden, die nicht nur menschenleer, sondern komplett leergefegt sind. Es zeichnet sich ab, dass dies die grösste Katastrophe ist seit Menschengedenken.

Alle Ärzte und Pflegerinnen und sämtliches Equipment, welches die Stiftung PanEco, die seit Jahren in Indonesien und speziell auch in Aceh arbeitet, nach Sumatra bringt, werden vor Ort und in Zusammenarbeit mit der YEL in der von dieser Organisation geleiteten Koordinationsstelle in Medan empfangen und in die örtlichen NGO-Teams integriert. Von dieser Zentrale aus werden all diese NGO-Helfer im Katastrophengebiet Nordsumatras koordiniert und gezielt eingesetzt, um die medizinische Versorgung und die Versorgung mit den wichtigsten Hilfsgütern aufzubauen.

Alle Bemühungen sind bisher jedoch ein Tropfen auf den heissen Stein: Es mangelt nicht am Willen der Menschen weltweit, mitzuhelfen und teilzunehmen beim Aufbau der dringend benötigten humanitären Hilfe, sondern an den Möglichkeiten.

Nach wie vor werden Ärztinnen und Ärzte, Pflegerinnen und Pfleger und medizinische Hilfsgüter gesucht, um eine erste Versorgung der Opfer zu ermöglichen. Und die Zeit läuft. 